\title{
Bound State Solutions of the Schrödinger Equation for the More General Exponential Screened Coulomb Potential Plus Yukawa (MGESCY) Potential Using Nikiforov-Uvarov Method
}

\author{
B. I. Ita1, H. Louis',2, O. U. Akakuru1, T. O. Magu', I. Joseph ${ }^{3}$, P. Tchoua ${ }^{4}$, P. I. Amos ${ }^{3}$, \\ I. Effiong ${ }^{5}$, N. A. Nzeata ${ }^{1}$
}

${ }^{1}$ Physical/Theoretical Chemistry Research Group, Department of Pure and Applied Chemistry, University of Calabar, CRS, Calabar, Nigeria

${ }^{2}$ CAS Key Laboratory for Nanosystem and Hierarchical Fabrication, CAS Centre for Excellence in Nanoscience, National Centre For Nanoscience and Technology, University of Chinese Academy of Science, Beijing, China

${ }^{3}$ Nano-Chemistry Research Group, Department of Chemistry, School of Physical Sciences,

Modibbo Adama University of Technology, Yola, Nigeria

${ }^{4}$ Department of Mathematics, Ngaoundere University, Ngaoundere, Cameroon

${ }^{5}$ Scientific Equipment Development Institute (SEDI), Enugu, Nigeria

Email: iserom2001@yahoo.com, Louis@nanoctr.cn

How to cite this paper: Ita, B.I., Louis, H., Akakuru, O.U., Magu, T.O., Joseph, I., Tchoua, P., Amos, P.I., Effiong, I. and Nzeata, N.A. (2018) Bound State Solutions of the Schrödinger Equation for the More General Exponential Screened Coulomb Potential Plus Yukawa (MGESCY) Potential using Nikiforov-Uvarov Method. Journal of Quantum Information Science, 8, 24-45.

https://doi.org/10.4236/jqis.2018.81003

Received: February 2, 2018

Accepted: March 27, 2018

Published: March 30, 2018

Copyright (c) 2018 by authors and Scientific Research Publishing Inc. This work is licensed under the Creative Commons Attribution International License (CC BY 4.0).

http://creativecommons.org/licenses/by/4.0/

\section{Abstract}

The solutions of the Schrödinger with more general exponential screened coulomb (MGESC), Yukawa potential (YP) and the sum of the mixed potential (MGESCY) have been presented using the Parametric Nikiforov-Uvarov Method (pNUM). The bound state energy eigenvalues and the corresponding un-normalized eigenfunctions expressed in terms of hypergeometric functions were obtained. Some derived equations were used to calculate numerical values for MGESC, YP, and MGESCY potentials for diatomic molecules with different screening parameters $(\alpha)$ for $I=0$ and $l=1$ state with $V_{0}=2.75 \mathrm{MeV}$ and $V_{1}=2.075 \mathrm{MeV}$. We observed an increase in 1 value; the particles behave more repulsive than attractive. The numerical values for different l-states at different screening parameters for CO molecules $(r=1.21282)$ and NO molecule ( $r=1.1508$ ) were obtained using the bound state energy eigenvalue of the Schrodinger equation for MGESC, YP and MGESCY potentials. Potential variation with intermolecular distance $(r)$ for some of the particles moving under the influence of MGESC, Yukawa and the mixed potential (MGESCY) were also studied. We also observed the variation of the MGESC potential 
with the radial distance of separation between the interacting particles $(r)$ for different screening parameters $(\alpha)$ with $V_{0}=2.75 \mathrm{MeV}$ at $l=0$ and $l=1$ and YP with $V_{1}=2.075 \mathrm{MeV}$ at $l=0$ and $l=1$ as purely diatomic particles in nature. The energies plotted against the principal quantum number $\mathrm{n}$ for different values of $(\alpha)$ for both $\mathrm{CO}$ and NO show closed resemblance even at different values of the potential depth. The energy plots of the YP and MGESC potential for both $\mathrm{CO}$ and NO molecules as $n \rightarrow \infty$, and the energy $E \rightarrow 0$, shows exothermal behaviour. The energy expression for the mixed potentials $V_{o}=5$ $\mathrm{MeV}$ and $V_{l}=10 \mathrm{MeV}$, shows that both diatomic molecules possesses similar behaviour.

\section{Keywords}

Yukawa, Exponential Screened Coulomb, Schrodinger, Hypergeometric

\section{Introduction}

The more general exponential screened coulomb (MGESC) potential expressed as

$$
V(r)=-\frac{V_{0}}{r}\left(1+(1+\alpha r) \mathrm{e}^{-2 \alpha r}\right)
$$

is a potential of great interest which on expansion comprises of the sum of coulomb potential, modified screened coulomb or the Yukawa potential and a modified exponential potential given as

$$
V(r)=-\frac{V_{0}}{r}-\frac{V_{0}}{r} \mathrm{e}^{-2 \alpha r}-V_{0} \alpha \mathrm{e}^{-2 \alpha r}
$$

This potential is known to describe adequately the effective potential of a many-body system of a variety of fields such as the atomic, solid state, plasma and quantum field theory [1]. The problem arising from screened coulomb potential is of indubitable importance in physics and chemistry of atomic incidence. To tackle this problem, various methods have been applied both numerically and analytically to obtain the energy spectrum of the particle under investigation. This method includes the WKB method [2], and various types of perturbation method [3] [4] [5]. The expansion of the MGESC Potential in Equation (1) gives

$$
V(r)=-\frac{V_{0}}{r}-\frac{V_{0}}{r} \sum_{i=0}^{\infty} V_{i}(\alpha r)^{i}
$$

The coefficient $V_{i}$ of Equation (3) can be obtained so that the perturbation method of [5] may be applied. Recently, a novel perturbative formalism which is based on decomposing the radial part of the Schrodinger equation into two having an Exact solvable part and approximate treatment depending on the nature of the perturbed potential [1] have been applied on the MGESC potential and bound state energies as well as wave functions to both bound and continuum region have been obtained. Hence, the Schrodinger equation for the MGESC Po- 
tential doesn't admit exact solution. For this reason, Sever and Tezcan [6] applied the large- $\mathrm{N}$ expansion following the method proposed by Mlodinow and Shatz [7] to obtain the energy eigenvalues for the ground state and the first excited state as well as their corresponding wavefunctions. Roy in 2013 [8] carried out extensive studies on some exponential screened coulomb potentials such as the Exponential Cosine Screened Coulomb (ECSC) and General Exponential Screened Coulomb (GESC) potential with special emphasis on higher states and stronger interactions. In his speculative studies, he obtained bound state solutions for both screened potentials via the Generalized Pseudospectral (GPS) method and computed reasonable results for the energy eigenvalues at different states compared with other results obtained in the literatures. Ita and Ekuri [9], carried out studies on the MGESC potential for diatomic molecules to obtain boundstate solutions of the Schrodinger equation using the Nikiforov-Uvarov (NU) Method.

The Yukawa potential, in atomics and particle physics expressed in the form

$$
V_{\text {yukawa }}(r)=-g^{2} \frac{\mathrm{e}^{-k m r}}{r} \equiv-\frac{V_{0}}{r} \mathrm{e}^{-\alpha r}
$$

where $g$ is the magnitude scaling constant, $m$ is the mass of the affected particle, $r$ is the radial distance to the particles, $k$ is another scaling constant which was first proposed by Hideki Yukawa in 1935on the paper titled "On the interaction of Elementary Particles" a work in which, he explained the effect of heavy nuclei interaction on pions. According to Yukawa, the interactions of particles is not always accompanied by emission of light particles when heavy particles are transmitted from neutron state to proton state, but the liberated energy due to the transmission is taken up sometimes by another heavy particles, which will be transformed from proton state into neutron state [10]. The Yukawa potential is a potential that decreases more rapidly with distance and can be expressed as the coulomb potential when $m \rightarrow 0$. Since then, numerous researches have been conducted by various scientists to obtain bound state of the potential by applying different scientific Methods. Gerry and Lamb in 1984 [11] studied the screened coulomb potential of the Yukawa type by using a scaling variational method based on the $\mathrm{SO}(2,1)$ Subgroup of the full $\mathrm{SO}(4.2)$ dynamic group of the point coulombic problem to obtain both energy eigenvalues for different states and Normalized wave functions. Gerry and Lamb, 1984 [11] applied the large-N phase Integral approximation based on the coherent states of $\mathrm{SO}(2,1)(\mathrm{SU}(1,1))$ to coulomb-like problems where they obtained energy eigenvalue for s-states of the Yukawa potential. Hamzavi and co-workers in 2012 [12] studied the Yukawa potential via a two body semi-relativistic (Spinless Salpeter) SS equation and obtained bound state energy values and their corresponding Normalized wavefunctions for short range Yukawa potential with arbitrary l-state using parametric NU Method. In their literature, it was spelt out that the known Static Screened Coulomb Potential (SSCP) yields reasonable results only for the innermost state when $Z$ is Large while it gives a rather poor result for the outermost and middle atomic states. Dutt et al., in 1984 [13], carried out studies on a 
screened coulomb potential by using a Rayleigh-Schrodinger Perturbation theory and obtained energy eigen values for large values of screening parameters. Their calculations to the energy eigenvalue yielded reasonable result compared to other numerical and analytical methods. Hamzavi and his colleagues in 2012 [14], applied the NU Method to the Yukawa potential for any l-state and obtained bound state approximate analytical solutions. Computed values for the bound state energy for different states were obtained and compared with the (Asymptotic Iteration Method) AIM, Supersymmetric (SUSY) and Numerical method as stated in their literature. Gerry and Lamb [15] obtained the energy eigenvalues for the Yukawa potential by using the Generalized Scaling Variational method for a system with a spherically symmetric columbic potential at the origin. The energy eigenvalues for different states and different screening parameters for bound states were obtained and these values were in agreement with those obtained in the literature Dutt and Varshni [13] studied the energy levels of neutral atoms by applying the shifted large-N expansion to the Yukawa potential with a modified screening parameter. They obtained energy values for the $\mathrm{k}$-shell over the range of atomic number $\mathrm{Z}$ up to 84 and compared with those obtained within the framework of hyper-viral-pad scheme they observed that the large- $\mathrm{N}$ techniques may also be applied in other areas of atomic physics. Sharma et al. [16] calculated bound state for all angular momenta for superposed two static screened coulomb potentials (SSCP) expressed as

$$
V(r)=-\left(g_{1} \mathrm{e}^{-\alpha r}+g_{2} \mathrm{e}^{-\gamma \alpha r}\right) / r
$$

where $g_{1}$ and $g_{2}$ are coupling constants, $\alpha$ is the screening parameter and $\gamma$ is the screening strength. By subjecting $g_{1}=0$, the modified screened coulomb potential as well as its numerical calculations for the bound state is obtained. Pakdel et al. [17] studied the Dirac equation with scalar and vector potential for the Yukawa potential and obtained both bound and scattering states. In their calculations, the energy eigenvalues for different values of $\mathrm{n}$ and $\mathrm{k}$ were reported numerically as well as their corresponding eigenstates. Since the screened coulomb potential plays significant role in microscopic fields, this potential has been applied in different branches of atomic and molecular physics and chemistry. For this reason, Roy [18] carried out studies on the critical parameters and spherical confinement of $\mathrm{H}$ atom in screened Coulomb potential using the GPS method. He extended his studies towards finding bound state energy eigenvalues for the screened coulomb potential

$$
V(r)=-\frac{V_{0}}{r} \sum_{i=0}^{\infty} V_{i}(\alpha r)^{i}
$$

and their corresponding wavefunctions as well as providing information regarding sample dipole polarizability. Onate and Ojunubah [19] applied the supersymmetric shape invariance approach and formalism on a class of YP expressed as 


$$
V(r)=\frac{-b r+r c \mathrm{e}^{-\alpha r}-a \mathrm{e}^{-2 \alpha r}}{r^{2}}
$$

and obtained bound state energy eigenvalue calculations. They deduced three different energy representations for the following potentials namely the coulomb, Yukawa and inversely quadratic Yukawa as they obtained their normalized wavefunctions and energy eigenvalues, compared to other related work via the NU and AP method in their literature and the values obtained yielded reasonable result. Ita et al. [20] obtained bound state solutions of the Schrödinger's equation for Manning-Rosen plus a Class of Yukawa (MRCY) potential given as

$$
V(r)=-\left[\frac{C \mathrm{e}^{-\alpha r}+D \mathrm{e}^{-2 \alpha r}}{\left(1-\mathrm{e}^{-\alpha r}\right)^{2}}\right]-\frac{V_{0} \mathrm{e}^{-\alpha r}}{r}-\frac{V_{0}^{\prime} \mathrm{e}^{-2 \alpha r}}{r^{2}}
$$

From their calculations, they deduced three different potentials such as the Manning-Rosen, Yukawa and inversely quadratic Yukawa potential and obtained bound state energy eigenvalues as well as wave functions for different principal quantum number $\mathrm{n}$ for the s-state. In view of the relativistic quantum mechanics, a particle moving in a potential field is described particularly with the Klein-Gordon (KG) equation. Over the years numerous works have been reported concerning the Klein-Gordon equations for various kinds of potentials by using different Methods such as supersymmetry [21], supersymmetric WKB approach [22], Nikiforov-Uvarov Method [23]-[30]. Ikhdair [31] obtained approximate analytical bound state solution of the Klein-Gordon equation with equal Scalar and Vector Eckart type potential given as

$$
V(r, q)=4 V_{1} \frac{\mathrm{e}^{-2 \alpha r}}{\left(1-q \mathrm{e}^{-2 \alpha r}\right)}-V_{2} \frac{1+q \mathrm{e}^{-2 \alpha r}}{\left(1-q \mathrm{e}^{-2 \alpha r}\right)}
$$

via the NU Method. Both energy equation as well as the un-normalized wave functions expressed in terms of the Jacobi polynomial were obtained. Ikot et al. [32] obtained approximate analytic solutions of the Klein-Gordon in D-dimension for any l-state for a seven parameter type potential expressed as

$$
V(r)=A+\frac{B}{\left(q+\mathrm{e}^{2 \alpha r}\right)}+\frac{C}{\left(q+\mathrm{e}^{2 \alpha r}\right)^{2}}+\frac{F b \mathrm{e}^{2 \alpha r}}{\left(q+\mathrm{e}^{2 \alpha r}\right)}+\frac{G b \mathrm{e}^{2 \alpha r}}{\left(q+\mathrm{e}^{2 \alpha r}\right)^{2}}
$$

where $A, B, C, F$ and $G$ are potential parameters, $q$ is the deformation parameter, $b=\mathrm{e}^{2 \alpha r_{e}}, r_{e}$ is the distance from equilibrium position and $\alpha$ is the screening parameter. In their work, they obtained both bound and scattering state with energy spectrum of some special potential such as Hulthen, Manning-Rosen, Eckart and Wood-Saxon potential. Hansabadi et al. [33] studied a special kratzer-type potential where $V(r) \neq S(r)$ and obtained bound state solution of the Klein-Gordon equation with position dependent mass $m(r)=m_{0}+\frac{m_{1}}{r}$ as well as the wavefunction.Since then many literatures have reported different 
special case of potential such as, Poschl-Teller potential [34], Rosen-Morse [16] and many more by applying different methods. Moreover when arbitrary angular momentum quantum number $l$ is present, one can only solve the SE and KGE approximately using suitable approximation scheme [35]. Some of such approximations include convectional approximation scheme proposed by Greene and Aldrich [36], improved approximation scheme [37], an elegant approximation scheme [38]. These approximations are used to deal with the centrifugal term or potential barrier arising from the problem of interest. Not much has been achieved on the Schrodinger equation for the MGESCY potential over the years. The aim of this report is to obtain bound state solutions of the Schrödinger equation for the More General Exponential Screened Coulomb Potential plus Yukawa (MGESCY) potential.

\section{Theoretical Approach}

The Nikiforov-Uvarov method is based on the solutions to a second-order linear differential equation with special orthogonal function [23]. The hyper-geometric type has been used to solve the Schrödinger, Klein-Gordon and Dirac equation for different kind of potentials [25]-[30].

\subsection{The More Generalized Form of Nikiforov-Uvarov Method}

Given a second order differential equation of the form

$$
\psi^{\prime \prime}(s)+\frac{\bar{\tau}(s)}{\sigma(s)} \psi^{\prime}(s)+\frac{\bar{\sigma}(s)}{\sigma^{2}(s)} \psi(s)=0
$$

In order to find the exact solutions to Equation (11), we set the wavefunction as

$$
\psi(s)=\phi(s) \chi(s)
$$

where $\phi(s)$ and $\chi(s)$ are the hypergeometric-type functions

And on substituting Equation (12) into Equation (11), then Equation (11) reduces to hypergeometric type

$$
\sigma(s) \chi^{\prime \prime}(s)+\tau(s) \chi^{\prime}(s)+\lambda \chi(s)=0
$$

where the wave function $\phi(s)$ is defined as the logarithmic derivative,

$$
\frac{\phi^{\prime}(s)}{\phi(s)}=\frac{\pi(s)}{\sigma(s)}
$$

where $\pi(s)$ is at most first order polynomials.

Likewise, the hypergeometric type function $\phi(s)$ in Equation (13) for a fixed $n$ is given by the Rodriques relation as

$$
\chi_{n}(s)=\frac{B_{n}}{\rho(S)} \frac{\mathrm{d}^{n}}{\mathrm{~d} s^{n}}\left[\sigma^{n}(s) \rho(s)\right]
$$

where $B_{n}$ is the normalization constant and the weight function $\rho(s)$ must satisfy the condition 


$$
\frac{\mathrm{d}}{\mathrm{d} s}\left[\sigma^{n}(s) \rho(s)\right]=\tau(s) \rho(s)
$$

with

$$
\tau(s)=\bar{\tau}(s)+2 \pi(s)
$$

In order to accomplish the condition imposed on the weight function $\rho(s)$, it is necessary that the classical or polynomials $\tau(s)$ be equal to zero to some point of an interval $(a, b)$ and its derivative at this interval at $\sigma(s)>0$ will be negative, that is

$$
\frac{\mathrm{d} \tau(s)}{\mathrm{d} s}<0 .
$$

Therefore, the function $\pi(s)$ and the parameters $\lambda$ required for the NU method are defined as follows:

$$
\begin{gathered}
\pi(s)=\frac{\sigma^{\prime}-\tilde{\tau}}{2} \pm \sqrt{\left(\frac{\sigma^{\prime}-\tilde{\tau}}{2}\right)^{2}-\bar{\sigma}+k \sigma} \\
\lambda=k+\pi^{\prime}(s)
\end{gathered}
$$

The s-values in Equation (19) are possible to evaluate if the expression under the square root be square of polynomials. This is possible, if and only if its discriminant is zero. With this, the new eigenvalues equation becomes

$$
\lambda=\lambda_{n}=-\frac{n \mathrm{~d} \tau}{\mathrm{d} s}-\frac{n(n-1)}{2} \frac{\mathrm{d}^{2} \sigma}{\mathrm{d} s^{2}}, n=0,1,2, \cdots
$$

On comparing Equation (20) and Equation (21), we can obtain the energy eigenvalues.

\subsection{Parametric Nikiforov-Uvarov Method}

The parametric form is simply using parameters to obtain explicitly energy eigenvalues and it is still based on the solutions of a generalized second order linear differential equation with special orthogonal functions. The hypergeometric NU method has shown high utility in calculating the exact energy levels of all bound states for some solvable quantum systems.

Given a second order differential equation of the form

$$
\psi^{\prime \prime}(s)+\frac{\bar{\tau}(s)}{\sigma(s)} \psi^{\prime}(s)+\frac{\bar{\sigma}(s)}{\sigma^{2}(s)} \psi(s)=0
$$

where $\sigma(s)$ and $\bar{\sigma}(s)$ are polynomials at most second degree and $\tilde{\tau}(s)$ is first degree polynomials. The parametric generalization of the $\mathrm{N}-\mathrm{U}$ method is given by the generalized hypergeometric-type equation

$$
\Psi^{\prime \prime}(s)+\frac{c_{1}-c_{2} s}{s\left(1-c_{3} s\right)} \Psi^{\prime}(s)+\frac{1}{s^{2}\left(1-c_{3} s\right)^{2}}\left[-\epsilon_{1} s^{2}+\epsilon_{2} s-\epsilon_{3}\right] \Psi(s)=0
$$

Thus Equation (22) can be solved by comparing it with Equation (23) and the following polynomials are obtained 


$$
\bar{\tau}(s)=\left(c_{1}-c_{2} s\right), \sigma(s)=s\left(1-c_{3} s\right), \bar{\sigma}(s)=-\epsilon_{1} s^{2}+\epsilon_{2} s-\epsilon_{3}
$$

The parameters obtainable from Equation (23) serve as important tools to finding the energy eigenvalue and eigenfunctions.

Now substituting Equation (24) into Equation (19):

$$
\bar{\sigma}(s)=c_{4}+c_{5} s \pm\left[\left(c_{6}-c_{3} k_{ \pm}\right) s^{2}+\left(c_{7}+k_{ \pm}\right) s+c_{8}\right]^{\frac{1}{2}}
$$

where

$$
\begin{aligned}
& c_{4}=\frac{1}{2}\left(1-c_{1}\right), c_{5}=\frac{1}{2}\left(c_{2}-2 c_{3}\right), c_{6}=c_{5}^{2}+\epsilon_{1}, c_{7}=2 c_{4} c_{5}-\epsilon_{2}, \\
& c_{8}=c_{4}^{2}+\epsilon_{3}, c_{9}=c_{3} c_{7}+c_{3}^{2} c_{8}+c_{6}
\end{aligned}
$$

The resulting value of $k$ in Equation (25) is obtained from the condition that the function under the square root is square of a polynomials and it yields,

$$
k_{ \pm}=-\left(c_{7}+2 c_{3} c_{8}\right) \pm 2 \sqrt{c_{8} c_{9}}
$$

The new $\pi(s)$ for $k_{-}$becomes

$$
\pi(s)=c_{4}+c_{5} s-\left[\left(\sqrt{c_{9}}+c_{3} \sqrt{c_{8}}\right) s-\sqrt{c_{8}}\right]
$$

for the $k_{-}$value,

$$
k_{-}=-\left(c_{7}+2 c_{3} c_{8}\right)-2 \sqrt{c_{8} c_{9}}
$$

Using Equation (17), we obtain

$$
\tau(s)=c_{1}+2 c_{4}-\left(c_{2}-2 c_{5}\right) s-2\left[\left(\sqrt{c_{9}}+c_{3} \sqrt{c_{8}}\right) s-\sqrt{c_{8}}\right] .
$$

The physical condition for the bound state solution is $\tau^{\prime}<0$ and thus

$$
\tau^{\prime}(s)=-2 c_{3}-2\left(\sqrt{c_{9}}+c_{3} \sqrt{c_{8}}\right)<0
$$

with the aid of Equations (20) and (21), we obtain the energy equation as

$$
\left(c_{2}-c_{3}\right) n+c_{3} n^{2}-(2 n+1) c_{5}+(2 n+1)\left(\sqrt{c_{9}}+c_{3} \sqrt{c_{8}}\right)+c_{7}+2 c_{3} c_{8}+2 \sqrt{c_{8} c_{9}}=0
$$

The weight function $\rho(s)$ is obtained from Equation (16) as

$$
\rho(s)=s^{c_{10}-1}\left(1-c_{3} s\right)^{\frac{c_{11}}{c_{3}}-c_{10}-1}
$$

and together with Equation (15), we have

$$
\chi_{n}(s)=P_{n}^{\left(c_{10}-1, \frac{c_{11}}{c_{3}}-c_{10}-1\right)}\left(1-2 c_{3} s\right)
$$

where

$$
c_{10}=c_{1}+2 c_{4}+2 \sqrt{c_{8}}, \quad c_{11}=c_{2}-2 c_{5}+2\left(\sqrt{c_{9}}+c_{3} \sqrt{c_{8}}\right)
$$

and $P_{n}^{(\alpha, \beta)}$ are the Jacobi polynomials. The second part of the wave function is obtained from Equation (14) as

$$
\phi(s)=s^{c_{12}}\left(1-c_{3} s\right)^{-c_{12}-\frac{c_{13}}{c_{3}}}
$$

where

$$
c_{12}=c_{4}+\sqrt{c_{8}}, \quad c_{13}=c_{5}-\left(\sqrt{c_{9}}+c_{3} \sqrt{c_{8}}\right)
$$


Thus, the total wave function becomes

$$
\psi(s)=N_{n} s^{c_{12}}\left(1-c_{3} s\right)^{-c_{12}-\frac{c_{13}}{c_{3}}} P_{n}^{\left(c_{10}-1, \frac{c_{11}}{c_{3}}-c_{10}-1\right)}\left(1-2 c_{3} s\right)
$$

where $N_{n}$ is the normalization constant.

\section{Bound State Solutions of Schrodinger Equation}

\subsection{Solutions to the Radial Part of the Schrodinger Equation with More General Exponential Screened Coulomb Potential (MGESCP)}

Given the radial Schrodinger equation as [26]

$$
\frac{\mathrm{d}^{2} R_{n l}(r)}{\mathrm{d} r^{2}}+\frac{2}{r} \frac{\mathrm{d} R_{n l}(r)}{\mathrm{d} r}+\frac{2 \mu}{\hbar^{2}}\left[E-V(r)-\frac{\lambda \hbar^{2}}{2 \mu r^{2}}\right] R_{n l}(r)=0,
$$

$\lambda=l(l+1)$ and $V(r)$ is the potential energy function given as

$$
V(r)=-\frac{V_{0}}{r}\left(1+(1+\alpha r) \mathrm{e}^{-2 \alpha r}\right)
$$

where $V_{0}$ is the potential depth of the MGESC potential and $\alpha$ is an adjustable positive parameter and takes any value between zero and infinity

$$
V_{\text {eff }}(r)=V(r)+\frac{l(l+1) \hbar^{2}}{2 \mu r^{2}}=-\frac{V_{0}}{r}\left(1+(1+\alpha r) \mathrm{e}^{-2 \alpha r}\right)+\frac{l(l+1) \hbar^{2}}{2 \mu r^{2}}
$$

Substituting the effective potential of Equation (41) into radial Schrodinger equation of Equation (39), we obtain

$$
\begin{aligned}
& \frac{\mathrm{d}^{2} R_{n l}(r)}{\mathrm{d} r^{2}}+\frac{2}{r} \frac{\mathrm{d} R_{n l}(r)}{\mathrm{d} r} \\
& +\frac{2 \mu}{\hbar^{2}}\left[E+\frac{V_{0}}{r}\left(1+(1+\alpha r) \mathrm{e}^{-2 \alpha r}\right)-\frac{l(l+1) \hbar^{2}}{2 \mu r^{2}}\right] R_{n l}(r)=0 \\
& \frac{\mathrm{d}^{2} R_{n l}(r)}{\mathrm{d} r^{2}}+\frac{2}{r} \frac{\mathrm{d} R_{n l}(r)}{\mathrm{d} r} \\
+ & \frac{2 \mu}{\hbar^{2}}\left[E+\frac{V_{0}}{r}+\frac{V_{0}}{r} \mathrm{e}^{-2 \alpha r}+V_{0} \alpha \mathrm{e}^{-2 \alpha r}-\frac{l(l+1) \hbar^{2}}{2 \mu r^{2}}\right] R_{n l}(r)=0 \\
\frac{\mathrm{d}^{2} R_{n l}(r)}{\mathrm{d} r^{2}}+\frac{2}{r} \frac{\mathrm{d} R_{n l}(r)}{\mathrm{d} r} & \frac{1}{r^{2}}\left[\frac{2 \mu}{\hbar^{2}}\left(E+V_{0} \alpha \mathrm{e}^{-2 \alpha r}\right) r^{2}+\frac{2 \mu}{\hbar^{2}}\left(V_{0}+V_{0} \mathrm{e}^{-2 \alpha r}\right) r-\gamma\right] R_{n l}(r)=0
\end{aligned}
$$

Introducing the following dimensional parameters,

$$
\begin{gathered}
-\beta^{2}=\frac{2 \mu}{\hbar^{2}}\left(E+V_{0} \alpha \mathrm{e}^{-2 \alpha r}\right) \\
\varepsilon=\frac{2 \mu}{\hbar^{2}}\left(V_{0}+V_{0} \mathrm{e}^{-2 \alpha r}\right) \\
\gamma=l(l+1) \\
\frac{\mathrm{d}^{2} R_{n l}(r)}{\mathrm{d} r^{2}}+\frac{2}{r} \frac{\mathrm{d} R_{n l}(r)}{\mathrm{d} r}+\frac{1}{r^{2}}\left[-\beta^{2} r^{2}+\varepsilon r-\gamma\right] R_{n l}(r)=0
\end{gathered}
$$


Comparing Equation (46) with Equation (32) yields the following dimensional parameters

$$
\begin{aligned}
\bar{\tau}(r) & =\left(c_{1}-c_{2} r\right)=2, \sigma(r)=r\left(1-c_{3} r\right)=r, \bar{\sigma}(r)=-\beta^{2} r^{2}+\varepsilon r-\gamma \\
c_{1} & =2, c_{2}=c_{3}=0, c_{4}=\frac{1}{2}\left(1-c_{1}\right)=-\frac{1}{2}, c_{5}=\frac{1}{2}\left(c_{2}-2 c_{3}\right)=0, \\
c_{6} & =c_{5}^{2}+\epsilon_{1}=\beta^{2}, c_{7}=2 c_{4} c_{5}-\epsilon_{2}=\varepsilon, c_{8}=c_{4}^{2}+\epsilon_{3}=\frac{1}{4}+\gamma, \\
c_{9} & =c_{3} c_{7}+c_{3}^{2} c_{8}+c_{6}=\beta^{2}
\end{aligned}
$$

Substituting the polynomial of Equation (47) into Equation (28), the following is obtained

$$
\begin{aligned}
\pi(r) & =\frac{1}{2} \pm \frac{1}{2} \sqrt{\frac{1}{4}+\beta^{2} r^{2}-\varepsilon r+\gamma+k r} \\
& =-\frac{1}{2} \pm \frac{1}{2} \sqrt{4 \beta^{2} r^{2}+4(k-\varepsilon) r+4 \gamma+1}
\end{aligned}
$$

The discriminant of the expression under the square root in Equation (49) has to be zero for it to have equal roots. Therefore, we obtain

$$
(4(k-\varepsilon))^{2}-4\left(4 \beta^{2}\right)(4 \gamma+1)=0
$$

On solving Equation (50), the following is obtained for

$$
k_{ \pm}=-\left(c_{7}+2 c_{3} c_{8}\right) \pm 2 \sqrt{c_{8} c_{9}}=-\varepsilon \pm \beta \sqrt{(4 \gamma+1)}
$$

where

$$
\begin{gathered}
k_{-}=-\varepsilon-\beta \sqrt{(4 \gamma+1)} \\
k_{+}=-\varepsilon+\beta \sqrt{(4 \gamma+1)}
\end{gathered}
$$

Substituting $k_{ \pm}$into Equation (49), gives the following four possible solutions obtained for $\pi(r)$ as

$$
\pi(r)=-\frac{1}{2} \pm \begin{cases}\beta r-\frac{1}{2} \sqrt{(4 \gamma+1)} & \text { for } k_{-}=-\varepsilon-\beta \sqrt{(4 \gamma+1)} \\ \beta r+\frac{1}{2} \sqrt{(4 \gamma+1)} & \text { for } k_{+}=-\varepsilon+\beta \sqrt{(4 \gamma+1)}\end{cases}
$$

From the four possible forms of $\pi(r)$ in Equation (34), we select the one for which the function $\tau(s)$ in Equation (19) has a negative derivative. $t(s)$ satisfies these requirements with:

$$
\tau(s)=1-2 \beta r+\sqrt{4 \gamma+1}
$$

and

$$
\tau^{\prime}(s)=-2 \beta<0
$$

Hence the new $\pi(r)$ for which $k_{-}$becomes

$$
\pi(r)=-\frac{1}{2}-\beta r+\frac{1}{2} \sqrt{(4 \gamma+1)}
$$




$$
\pi^{\prime}(r)=-\beta
$$

From Equation (20),

$$
\lambda=k+\pi^{\prime}(s)=-\varepsilon-\beta \sqrt{(4 \gamma+1)}-\beta
$$

and also from Equation (21),

$$
\lambda=\lambda_{n}=-\frac{n \mathrm{~d} \tau}{\mathrm{d} s}-\frac{n(n-1)}{2} \frac{\mathrm{d}^{2} \sigma}{\mathrm{d} s^{2}}=2 n \beta \quad(n=0,1,2, \cdots)
$$

solving Equation (57) and Equation (58) explicitly, we obtain

$$
\beta^{2}=\left(\frac{\varepsilon}{2 n+1+\sqrt{(4 \gamma+1)}}\right)^{2}
$$

Substituting the values of $\beta^{2}, \varepsilon$ and $\gamma$ of Equation (45) into Equation (59) yield

$$
E_{n, l}=-V_{0} \alpha \mathrm{e}^{-2 \alpha r}-\frac{\mu}{2 \hbar^{2}}\left(\frac{\left(V_{0}+V_{0} \mathrm{e}^{-2 \alpha r}\right)}{n+l+1}\right)^{2}
$$

To obtain the radial wave function o Equation (3), where $c_{3} \rightarrow 0$, the following expressions are obtained

$$
\begin{gathered}
\chi_{n}(r)=L_{n}^{c_{10}-1}\left(c_{11} r\right) \\
\phi(r)=r^{c_{12}} \mathrm{e}^{c_{13} r}
\end{gathered}
$$

where

$$
\begin{gathered}
\lim _{c_{3} \rightarrow 0} P_{n}^{\left(c_{10}-1, \frac{c_{11}}{c_{3}}-c_{10}-1\right)}\left(1-2 c_{3} r\right)=L_{n}^{c_{10}}\left(c_{11} r\right) \\
\lim _{c_{3} \rightarrow 0}\left(1-c_{3} r\right)^{-c_{12}-\frac{c_{13}}{c_{3}}}=\mathrm{e}^{c_{13} s}
\end{gathered}
$$

Then, the radial wave function can be expressed

$$
R_{n l}(r)=N_{n} r^{c_{12}} \mathrm{e}^{c_{13} r} L_{n}^{c_{10}}\left(c_{11} r\right)
$$

where

$$
c_{10}=1+\sqrt{4 l(l+1)+1}, c_{12}=-\frac{1}{2}+\frac{1}{2} \sqrt{4 l(l+1)+1}, c_{13}=-\beta, c_{11}=2 \beta
$$

Then the wave functions for the MGESC potential is expressed below as

$$
\begin{aligned}
R_{n l}(r) & =N_{n} r^{-\frac{1}{2}+\frac{1}{2} \sqrt{4 l(l+1)+1}} \mathrm{e}^{-\beta r} L_{n}^{1+\sqrt{4 l(l+1)+1}}(2 \beta r) \\
\text { if } r & =(2 \beta)^{-1} v \text { and } \alpha=\frac{1}{2} \sqrt{4 l(l+1)+1}
\end{aligned}
$$

Substituting Equation (65) into Equation (64), we obtain

$$
R_{n l}(r)=N_{n, l}(2 \beta)^{\frac{1}{2}-\alpha} v^{-\frac{1}{2}+\alpha} \mathrm{e}^{-\frac{v}{2}} L_{n}^{1+2 \alpha}(v)
$$

where $N_{n, l}$ is the normalization constant. 


\subsection{Solutions to the Radial Part of the Schrodinger Equation with the Yukawa Potential}

Given the radial Schrodinger equation as

$$
\frac{\mathrm{d}^{2} R_{n l}(r)}{\mathrm{d} r^{2}}+\frac{2 \mu}{\hbar^{2}}\left[E-V_{\text {eff }}(r)\right] R_{n l}(r),
$$

where $V_{\text {eff }}(r)$ is the effective potential energy function given as

$$
V(r)=-\frac{V_{1} \mathrm{e}^{-\alpha r}}{r}+\frac{l(l+1) \hbar^{2}}{2 \mu r^{2}},
$$

$V_{1}$ s the potential depth of the YP and $\alpha$ is an adjustable positive parameter. Inserting Equation (68) into Equation (67), we obtain

$$
\frac{\mathrm{d}^{2} R_{n l}(r)}{\mathrm{d} r^{2}}+\frac{2 \mu}{\hbar^{2}}\left[E+\frac{V_{1} \mathrm{e}^{-\alpha r}}{r}-\frac{l(l+1) \hbar^{2}}{2 \mu r^{2}}\right] R_{n l}(r),
$$

Equation (69) cannot be solved exactly for $l \neq 0$ hence to overcome this barrier, we introduce an approximation of the pekeris type for the centrifugal [24] [25] [26] [27] term as

$$
\frac{1}{r^{2}}=\frac{\alpha^{2}}{\left(1-\mathrm{e}^{-\alpha r}\right)^{2}} ; \frac{1}{r}=\frac{\alpha}{\left(1-\mathrm{e}^{-\alpha r}\right)}
$$

making the transformation $s=\mathrm{e}^{-\alpha r}$ Equation (68) becomes

$$
V(s)=-\frac{V_{1} \alpha s}{(1-s)}+\frac{l(l+1) \hbar^{2} \alpha^{2}}{2 \mu(1-s)^{2}}
$$

Again, applying the transformation $s=\mathrm{e}^{-\alpha r}$ to get the form that NU method is applicable, Equation (67) gives a generalized hypergeometric-type equation as

$$
\begin{aligned}
& \frac{\mathrm{d}^{2} R(s)}{\mathrm{d} s^{2}}+\frac{(1-s)}{(1-s) s} \frac{\mathrm{d} R(s)}{\mathrm{d} s} \\
& +\frac{1}{(1-s)^{2} s^{2}}\left[-\left(\beta^{2}+B\right) s^{2}+\left(2 \beta^{2}+B\right) s-\left(\beta^{2}+\lambda\right)\right] R(s)=0
\end{aligned}
$$

where

$$
-\beta^{2}=\frac{2 \mu E}{\alpha^{2} \hbar^{2}}, B=\frac{2 \mu V_{1}}{\alpha \hbar^{2}}
$$

Comparing Equation (71) with Equations (32), (26), (35) and (37) yields the following parameters

$$
\begin{aligned}
& c_{1}=c_{2}=c_{3}=1, c_{4}=0, c_{5}=-\frac{1}{2}, c_{6}=\frac{1}{4}+\beta^{2}+B, c_{7}=-2 \beta^{2}-B, \\
& c_{8}=\beta^{2}+\lambda, c_{9}=\frac{1}{4}+\lambda, c_{10}=1+2 \sqrt{\beta^{2}+\lambda}, c_{11}=2+2\left(\sqrt{\frac{1}{4}+\lambda}+\sqrt{\beta^{2}+\lambda}\right) \\
& c_{12}=\sqrt{\beta^{2}+\lambda}, c_{13}=-\frac{1}{2}-\left(\sqrt{\frac{1}{4}+\lambda}+\sqrt{\beta^{2}+\lambda}\right), \\
& \epsilon_{1}=\beta^{2}+B, \epsilon_{2}=2 \beta^{2}+B, \epsilon_{3}=\beta^{2}+\lambda
\end{aligned}
$$


Now using Equations (32), (72) and (73) we obtain the energy eigen spectrum of the YP as

$$
\beta^{2}=\left[\frac{2 \lambda-B+\left(n^{2}+n+\frac{1}{2}\right)+(2 n+1) \sqrt{\frac{1}{4}+\lambda}}{(2 n+1)+2 \sqrt{\frac{1}{4}+\lambda}}\right]^{2}-\lambda,
$$

Equation (74) can be solved explicitly and the energy eigen spectrum of YP becomes

$$
E=-\frac{2 \mu}{\hbar^{2}}\left[\frac{V_{1}}{2(n+l+1)}-\frac{\alpha \hbar^{2}(n+l+1)}{2 \mu}\right]^{2}
$$

We now calculate the radial wave function of the YP as follows:

Using Equation (73), the weight function $\rho(s)$ of Equation (33) is given as

$$
\rho(s)=s^{2 U}(1-s)^{2 V},
$$

where $U=\sqrt{\beta^{2}+\lambda}$ and $V=\sqrt{\frac{1}{4}+\lambda}$

Also we obtain the wave function $\chi(s)$ as

$$
\chi(s)=P_{n}^{(2 U, 2 V)}(1-2 s)
$$

where $P_{n}^{(2 U, 2 V)}$ are Jacobi polynomials

Lastly,

$$
\varphi(s)=s^{c_{12}}\left(1-c_{3} s\right)^{-c_{12}-\frac{c_{13}}{c_{3}}},
$$

And using Equation (14) we get

$$
\varphi(s)=s^{U}(1-s)^{\frac{1}{2}+V},
$$

we then obtain the radial wave function from the equation

$$
R_{n}(s)=N_{n} s^{U}(1-s)^{\frac{1}{2}+V} P_{n}^{(2 U, 2 V)}(1-2 s),
$$

where $n$ is a positive integer and $N_{n}$ is the normalization constant.

\subsection{Solutions to the Radial Part of the Schrodinger Equation with More General Exponential Screened Coulomb plus Yukawa (MGESCY) Potential}

Combining the potential of Equation (40) and the Yukawa potential of Equation (4), we obtain

$$
V(r)=-\frac{V_{0}}{r}\left(1+(1+\alpha r) \mathrm{e}^{-2 \alpha r}\right)-\frac{V_{1} \mathrm{e}^{-\alpha r}}{r}
$$

Considering the MGESCY potential expression of Equation (81), on substitution into Equation (39) given as 


$$
\begin{aligned}
& \frac{\mathrm{d}^{2} R_{n l}(r)}{\mathrm{d} r^{2}}+\frac{2}{r} \frac{\mathrm{d} R_{n l}(r)}{\mathrm{d} r} \\
& +\frac{2 \mu}{\hbar^{2}}\left[E+\frac{V_{0}}{r}\left(1+(1+\alpha r) \mathrm{e}^{-2 \alpha r}\right)+\frac{V_{1}}{r} \mathrm{e}^{-\alpha r}-\frac{l(l+1) \hbar^{2}}{2 \mu r^{2}}\right] R_{n l}(r)=0
\end{aligned}
$$

we obtained both bound state solution as well as un-normalized wave function of the Schrodinger equation after solving Equation (82) explicitly by applying the NU method as

$$
E_{n, l}=-V_{0} \alpha \mathrm{e}^{-2 \alpha r}-\frac{\mu}{2 \hbar^{2}}\left(\frac{\left(V_{0}+V_{0} \mathrm{e}^{-2 \alpha r}+V_{1} \mathrm{e}^{-\alpha r}\right)}{n+l+1}\right)^{2}
$$

And the radial wave function expressed as

$$
\begin{gathered}
R_{n l}(r)=N_{n} r^{-\frac{1}{2}+\frac{1}{2} \sqrt{4 l(l+1)+1}} \mathrm{e}^{-\beta r} L_{n}^{1+\sqrt{4(l+1)+1}}(2 \beta r) \\
\text { if } r=(2 \beta)^{-1} v \text { and } \alpha=\frac{1}{2} \sqrt{4 l(l+1)+1}
\end{gathered}
$$

substituting Equation (85) into Equation (84), we obtain

$$
R_{n l}(r)=N_{n, l}(2 \beta)^{\frac{1}{2}-\alpha} v^{-\frac{1}{2}+\alpha} \mathrm{e}^{-\frac{v}{2}} L_{n}^{1+2 \alpha}(v)
$$

where $N_{n, l}$ is the normalization constant.

\section{Numerical Analysis}

The aim of this report is to obtain both bound state and their corresponding eigenfunctions of the Schrodinger for the Mixed Potential (MGESCY) potential. To fulfil this aim, we now use some of the previously derived equations to calculate numerical values for the MGESC potential, Yukawa potential and also the sum of both potential known as the MGESCY potential for diatomic molecules with different screening parameters $\alpha$ for $l=0$ and $l=1$ state using python program.

\subsection{Numerical Values}

Considering the bound state energy eigenvalue equation expressed in Equation (60), we obtained numerical values for different l-states at different screening parameters for CO molecule $(r=1.21282)$ and NO molecule $(r=1.1508)$ as shown in Tables 1-6.

To obtain the bound state energy eigen values of the Yukawa potential for diatomic molecules, we considered Equation (83) by subjecting $V_{0}=0$ for two states

For $\mathrm{CO}$ and NO diatomic molecules moving under the influence of the mixed potential, we obtained the Energy eigenvalues given in Equation (83) and computed numerical values for different screening parameter.

The $r$ values for $\mathrm{N}_{2}$ (1.0940), $\mathrm{CO}$ (1.21282) and $\mathrm{NO}$ (1.1508) were adapted from M. Karplus and R. N. Porter, Atoms and Molecules [39]. 
Table 1. Bound State Energy eigenvalues of the MGESC potential at $\alpha=0.01$ and $\alpha=0.03, \quad l=0$.

\begin{tabular}{ccccccc}
\hline \multicolumn{3}{c}{$\alpha=0.01$} & \multicolumn{3}{c}{$\alpha=0.03$} \\
\cline { 2 - 7 }$n$ & $\mathrm{CO}$ & $\mathrm{NO}$ & $\mathrm{CO}$ & $\mathrm{NO}$ \\
\cline { 2 - 7 } & $V_{0}=2.75 \mathrm{MeV}$ & $V_{0}=2.75 \mathrm{MeV}$ & $V_{0}=5 \mathrm{MeV}$ & $V_{0}=10 \mathrm{MeV}$ & $V_{0}=5 \mathrm{MeV}$ & $V_{0}=10 \mathrm{MeV}$ \\
\hline \multirow{2}{*}{1} & -3.7180178 & -3.7225783 & -11.777558 & -46.8312884 & -11.8199261 & -46.9997199 \\
2 & -1.6673640 & -1.6694094 & -5.3119550 & -20.9668751 & -5.3310739 & -21.0443114 \\
3 & -0.9496352 & -0.9508002 & -3.0489938 & -11.9170305 & -3.05997575 & -11.9599184 \\
4 & -0.6174293 & -0.6181869 & -2.0015661 & -7.7273196 & -2.00878169 & -7.7551422 \\
5 & -0.4369717 & -0.4375080 & -1.4325929 & -5.45142726 & -1.43776271 & -5.4710662 \\
6 & -0.3281615 & -0.3285644 & -1.0895201 & -4.07913595 & -1.09345627 & -4.0934052 \\
7 & -0.2575395 & -0.2578557 & -0.6685268 & -3.1884661 & -0.86998815 & -3.1996031 \\
8 & -0.2091213 & -0.2093782 & -0.7141926 & -2.57782580 & -0.71677913 & -2.5713199 \\
\hline
\end{tabular}

Table 2. Bound State Energy eigenvalues of the MGESC potential for $\alpha=0.03$ at $l=1$.

\begin{tabular}{|c|c|c|c|c|}
\hline \multirow{3}{*}{$n$} & \multicolumn{4}{|c|}{$\alpha=0.03$} \\
\hline & \multicolumn{2}{|c|}{$\mathrm{CO}$} & \multicolumn{2}{|c|}{ NO } \\
\hline & $V_{0}=5 \mathrm{MeV}$ & $V_{0}=10 \mathrm{MeV}$ & $V_{0}=5 \mathrm{MeV}$ & $V_{0}=10 \mathrm{MeV}$ \\
\hline 1 & -5.4720184 & -21.7904705 & -5.4787305 & -21.8171974 \\
\hline 2 & -3.0993611 & -12.2998410 & -3.1031632 & -12.3149287 \\
\hline 3 & -2.0011597 & -7.9070355 & -2.0036148 & -7.9167348 \\
\hline 4 & -1.4046059 & -5.5202019 & -1.4063294 & -5.5275928 \\
\hline 5 & -1.0449027 & -4.0820076 & -1.0461850 & -4.0701559 \\
\hline 6 & -0.8114416 & -3.1481629 & -0.8124375 & -3.1520255 \\
\hline 7 & -0.6513814 & -2.5079220 & -0.6521810 & -2.5109943 \\
\hline 8 & -0.5369127 & -2.0499615 & -0.3755048 & -2.0524772 \\
\hline
\end{tabular}

Table 3. Bound State Energy eigenvalues of the yukawa potential at $\alpha=0.01$ at $l=0$.

\begin{tabular}{lllll}
\hline & \multicolumn{3}{c}{$\alpha=0.01$} \\
\cline { 3 - 4 } & \multicolumn{3}{c}{$\mathrm{CO}$} & $\mathrm{NO}$ \\
\cline { 2 - 5 } & $V_{1}=2.075 \mathrm{MeV}$ & $V_{1}=5 \mathrm{MeV}$ & $V_{1}=5 \mathrm{MeV}$ \\
\hline 1 & -0.5253053 & -3.0501106 & -0.5259573 & -3.0538963 \\
2 & -0.2334690 & -1.3556047 & -0.2337588 & -1.3572873 \\
3 & -0.1313263 & -0.7625276 & -0.1314893 & -0.7634741 \\
4 & -0.0840488 & -0.4881771 & -0.0841531 & -0.4886234 \\
5 & -0.0536725 & -0.3389011 & -0.0584397 & -0.3393218 \\
6 & -0.0422066 & -0.2489886 & -0.0429353 & -0.2492976 \\
7 & -0.0323158 & -0.1906319 & -0.0328723 & -0.1908685 \\
\hline
\end{tabular}


Table 4. Bound State Energy eigenvalues of the yukawa potential at $\alpha=0.01$ at $l=1$.

\begin{tabular}{|c|c|c|c|c|}
\hline \multirow{3}{*}{$n$} & \multicolumn{4}{|c|}{$\alpha=0.01$} \\
\hline & \multicolumn{2}{|c|}{$\mathrm{CO}$} & \multicolumn{2}{|c|}{ NO } \\
\hline & $V_{1}=2.075 \mathrm{MeV}$ & $V_{1}=5 \mathrm{MeV}$ & $V_{1}=2.075 \mathrm{MeV}$ & $V_{1}=5 \mathrm{MeV}$ \\
\hline 1 & -0.2334690 & -1.3556047 & -0.2337588 & -1.3572872 \\
\hline 2 & -0.1313263 & -0.7625276 & -0.1314893 & -0.7634740 \\
\hline 3 & -0.0840488 & -0.4880177 & -0.0841531 & -0.4886234 \\
\hline 4 & -0.0583673 & -0.3389011 & -0.0584397 & -0.3393218 \\
\hline 5 & -0.0428821 & -0.2489806 & -0.0429352 & -0.2492976 \\
\hline 6 & -0.0328316 & -0.1906319 & -0.0328723 & -0.1908685 \\
\hline 7 & -0.0259410 & -0.1506227 & -0.0259732 & -0.1500969 \\
\hline 8 & -0.0211221 & -0.1220044 & -0.0210329 & -0.1221555 \\
\hline
\end{tabular}

Table 5. Bound state energy eigenvalues of the mixed potential at $l=0, \alpha=0.01$ and $\alpha=0.03, V_{0}=5 \mathrm{MeV}, V_{1}=10 \mathrm{MeV}$.

\begin{tabular}{|c|c|c|c|c|}
\hline \multirow{2}{*}{$n$} & \multicolumn{2}{|c|}{$\alpha=0.01$} & \multicolumn{2}{|c|}{$\alpha=0.03$} \\
\hline & $\mathrm{CO}$ & NO & $\mathrm{CO}$ & NO \\
\hline 1 & -48.8541621 & -48.9144403 & -46.6610242 & -46.8319028 \\
\hline 2 & -21.7400730 & -21.7668970 & -20.1571763 & -20.9195255 \\
\hline 3 & -12.2501418 & -12.2652568 & -11.7696030 & -11.8129699 \\
\hline 4 & -7.5765943 & -7.6735482 & -7.5292080 & -7.6100979 \\
\hline 5 & -5.4716195 & -5.4737100 & -5.3085336 & -5.3279823 \\
\hline 6 & -4.0329128 & -4.0379117 & -3.9371500 & -3.9515762 \\
\hline 7 & -3.0991367 & -3.1029609 & -3.0470693 & -3.0582366 \\
\hline 8 & -2.4594302 & -2.4619773 & -2.4368832 & -2.4457656 \\
\hline
\end{tabular}

Table 6. Bound State Energy eigenvalues for $0 \leq \alpha \leq 0.05$ for different State of Diatomic Molecules in Atomic unit $\hbar=\mu=\alpha=1$; $V_{0}=2.75 \mathrm{MeV}, V_{1}=2.075 \mathrm{MeV}$.

\begin{tabular}{ccccccc}
\hline \multirow{3}{*}{$n, I$} & \multicolumn{3}{c}{$\alpha=0.01$} & & \multicolumn{2}{c}{$\alpha=0.03$} \\
\cline { 2 - 6 } & $\mathrm{N}_{2}$ & $\mathrm{CO}$ & $\mathrm{NO}$ & $\mathrm{N}_{2}$ & $\mathrm{CO}$ & NO \\
\hline 1.0 & -7.04486 & -7.02827 & -7.03692 & -6.79940 & -6.75229 & -6.77682 \\
2.0 & -3.14596 & -3.13859 & -3.14245 & -3.06487 & -3.04363 & -3.05469 \\
2.1 & -1.78139 & -1.77200 & -1.77938 & -1.75779 & -1.74560 & -1.75195 \\
3.0 & -1.78139 & -1.77200 & -1.77938 & -1.75779 & -1.74560 & -1.75195 \\
3.1 & -1.14977 & -1.14707 & -1.14848 & -1.15280 & -1.14480 & -1.14896 \\
3.2 & -0.80667 & -0.80477 & -0.80576 & -0.82416 & -0.81844 & -0.82142 \\
\hline
\end{tabular}

\subsection{Potential Variation}

We start by giving an overview of the differences in the effective potential plots against internuclear distance of some particles moving under the influence of the 
MGESC potential in Figures 1-3 and the Yukawa potential in Figure 4 and Figure 5 . We have set $l=0$, excluding the angular momentum quantum number in Figure 1 and Figure 4 for the MGESC potential and Yukawa potential respectively, at $\alpha=0.01, \alpha=0.03, \alpha=2$ and $\alpha=5$. Also, we chose $l=1$ in Figure 2 and Figure 5, and $l=2$ in Figure 3 and obtained plots that describes particles which are diatomic in nature. The different plots represented below simply show how energy varies with distance for different values of the screening potential. In Figure 6, we have shown the variation of the effective potential (MGESCY) potential at $\alpha=0.01$, for $l=0$ and $l=1$.

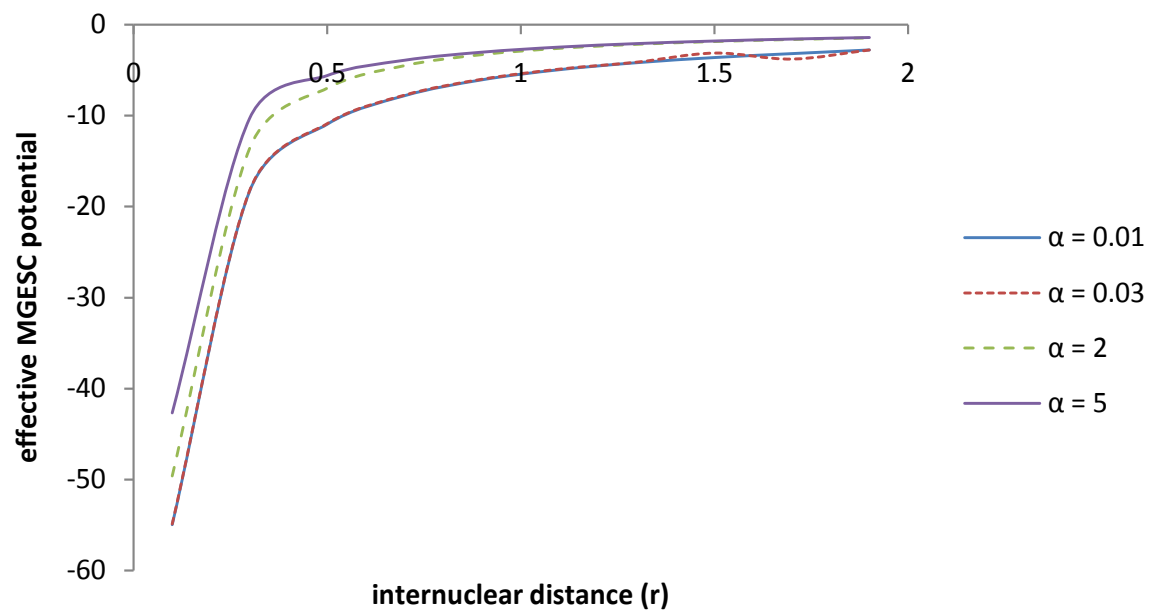

Figure 1. Variation of the effective MGESC potential against distance $(r)$ for different $\alpha$ values at $l=0, V_{0}=2.75 \mathrm{MeV}$.

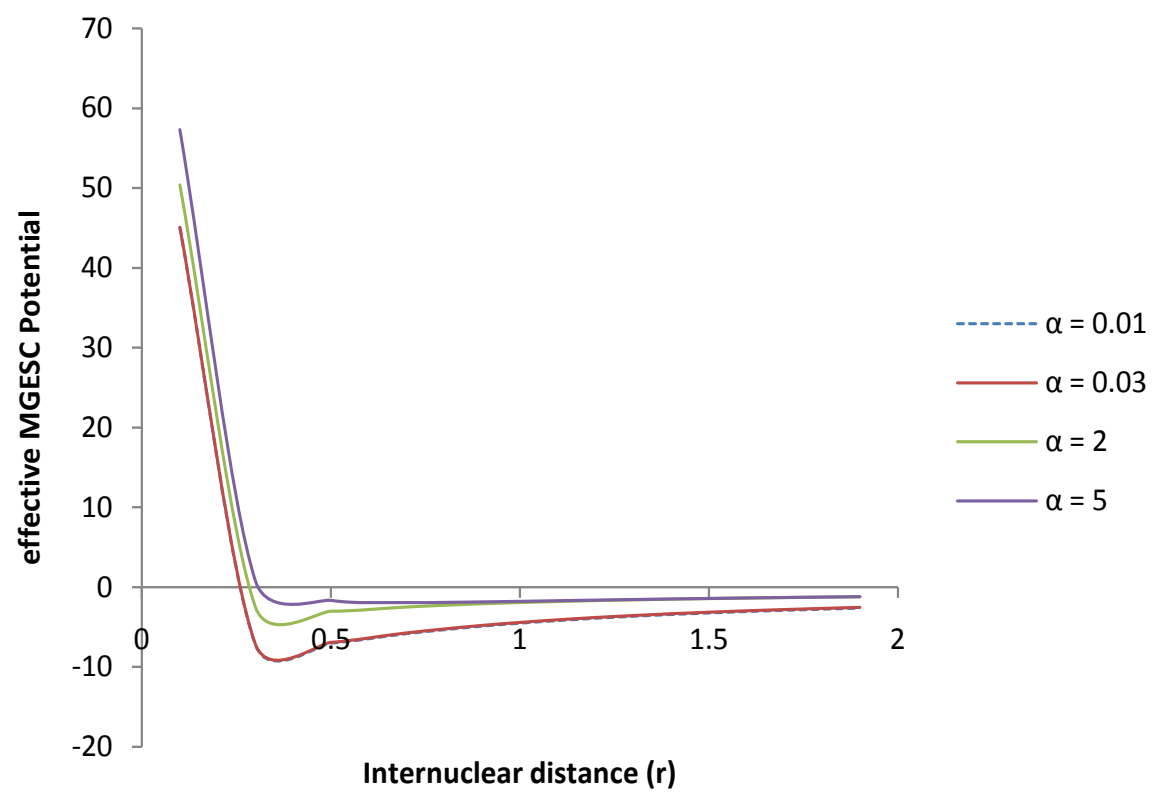

Figure 2. Variation of the effective MGESC potential against distance for different $\alpha$ values at $l=1, V_{0}=2.75 \mathrm{MeV}$. 


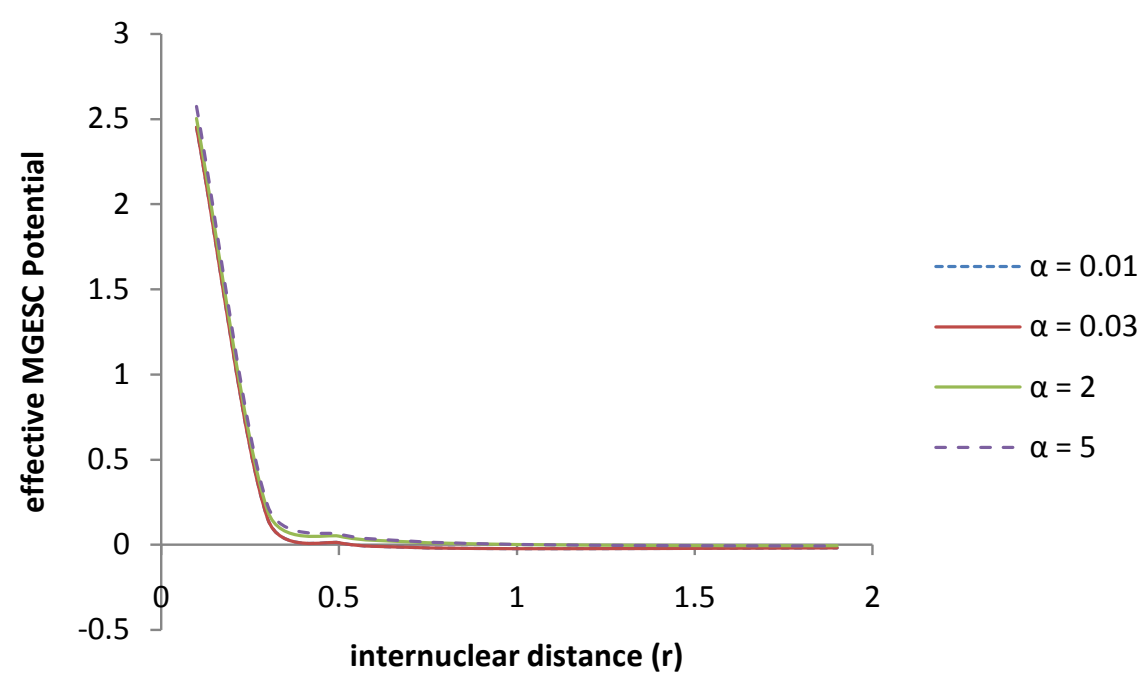

Figure 3. Variation of the effective MGESC potential against distance for different $\alpha$ values at $l=2, V_{0}=2.75 \mathrm{MeV}$.

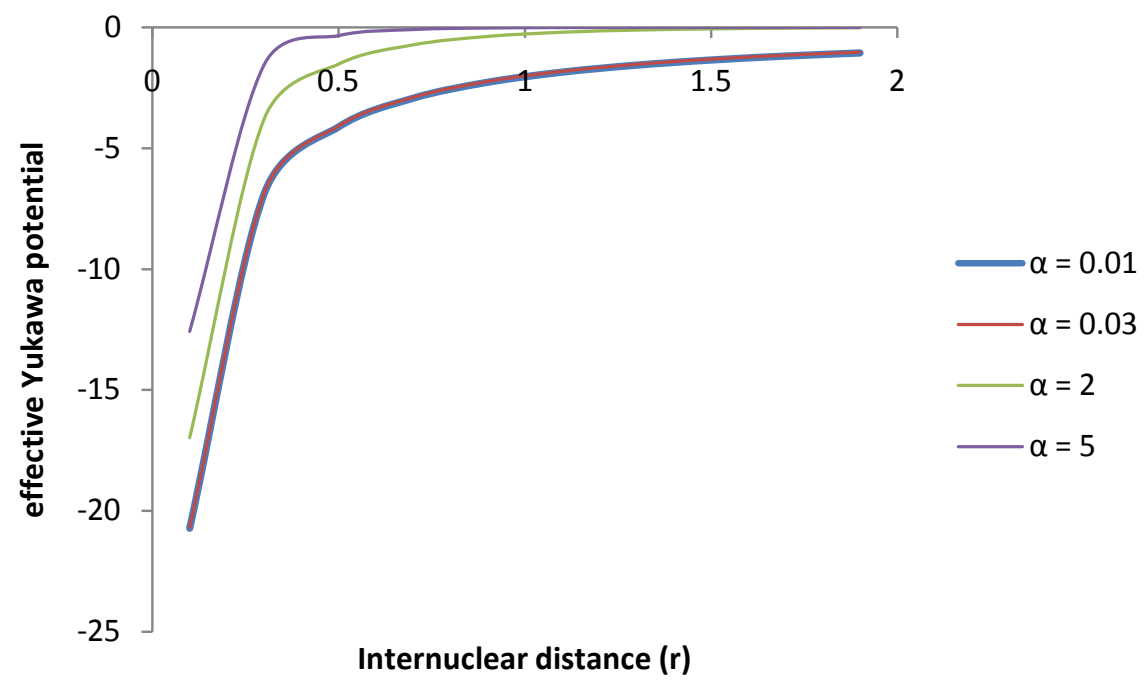

Figure 4. Variation of effective Yukawa potential against distance for different $\alpha$ values at $l=0, V_{1}=2.075 \mathrm{MeV}$.

\section{Discussions}

The variation of the MGESC potential with the radial distance of separation between the interacting particles $(r)$ for different screening parameters $(\alpha)$ with $V_{0}=2.75 \mathrm{MeV}$ at $l=0$ and $l=1$ in Figures $1-3$ and the Yukawa potential with $V_{1}=2.075 \mathrm{MeV}$ at $l=0$ and $l=1$ in Figure 4 and Figure 5 gives a full description of the behaviour of particles that are purely diatomic. When the 1 value increases, keeping the potential strength constant, the particles behave more repulsive than attractive. We tested the energy obtained in the Equations (60) and (83) for two diatomic molecules ( $\mathrm{CO}$ and $\mathrm{NO}$ ) and obtained results presented in Tables 1-6. The energies plot against $\mathrm{n}$ for different values of $(\alpha)$ for both $\mathrm{CO}$ and NO show close resemblance even at different values of the potential depth. 


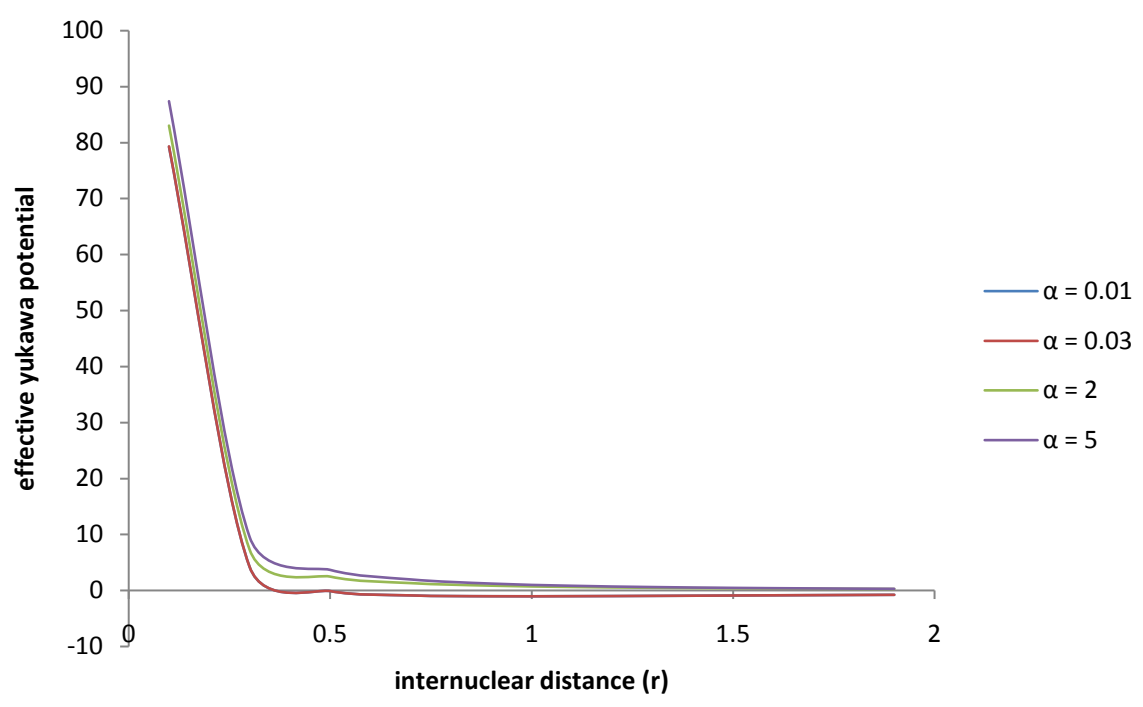

Figure 5. Variation of effective Yukawa potential for different $a$ values at $l=1$, $V_{1}=2.075 \mathrm{MeV}$.

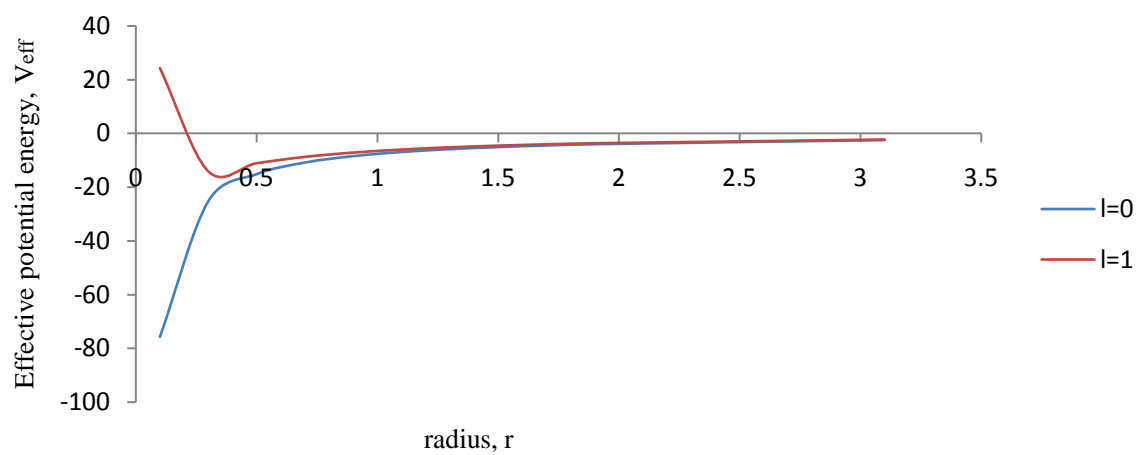

Figure 6. More general exponential screened coulomb plus Yukawa (MGESCY) potential versus $r$ with $V_{0}=2.75 \mathrm{MeV}, V_{0}=2.75 \mathrm{MeV}$ and $\alpha=0.01$ for $l=0$ and $l=1$.

Comparing the energy plots of the Yukawa and the MGESC potential for both $\mathrm{CO}$ and NO molecules, one can see that as $n \rightarrow \infty$, the energy obtained $E \rightarrow 0$, which describes exothermal behaviour (supporting information). For the mixed potentials where $V_{0}=5 \mathrm{MeV}$ and $V_{1}=10 \mathrm{MeV}$, the energy expression in Table 5 shows that both diatomic molecules possesses similar behaviour.

\section{Conclusion}

The analytical solutions of both Schrodinger for the more general exponential screened coulomb plus Yukawa (MGESCY) potential have been presented via the NU method. The Nikiforov-Uvarov (NU) method employed in the solutions enables us to explore an effective way of obtaining the energy eigenvalues and their corresponding eigenfunctions of the Schrödinger equations for any l-state. Finally, we calculate the energies and also obtained graphs of the MGESC potential, Yukawa potential and the mixed potential for diatomic molecules by means of Equations (60) and (83), for the l-states at different values of the screened pa- 
rameters (Supporting information). The explicit values of the energy at different values of the screened parameter are shown in Tables 1-6.

\section{Acknowledgements}

The authors greatly appreciate the effort of Professor Benedict IseromIta and Nelson Nzeata-Ibe and other colleagues in Mathematics and Computer Science Department, University of Calabar, Nigeria for their relevant scientific input.

\section{References}

[1] Ikhdair, S.M. and Sever, R. (2006) Bound State of a More General Exponential Screened Coulomb Potential. Journal of Mathematical Chemistry, 41, 343-353.

[2] Foldy, L.L. (1958) Fermi-Segrè Formula. Physical Review, 111, 1093-1098.

[3] Martin, I. and Simons, G. (1975) New Procedure for Generating Valence and Rydberg Orbitals. II. Atomic Photoionization Cross Sections. The Journal of Chemical Physics, 62, 4799-4803.

[4] Pratt, R.H. and Tseng, H.K. (1972) Behavior of Electron Wave Function near Te Nucleus and Normalization Screening Theory in the Atomic Photo Effect. Physical Review A, 5, 1063-1072. https://doi.org/10.1103/PhysRevA.5.1063

[5] Kratzer, S. and Frey, J. (1978) Transient Velocity Characteristics of Electrons in GaAs with $\Gamma$-L-Xconduction Band Ordering. Physical Review A, 13, 532-541.

[6] Sever, R. and Tezcan, C. (1987) 1/N Expansion for a More General Screened Coulomb Potential. Physical Review A, 6, 1045-1049.

https://doi.org/10.1103/PhysRevA.36.1045

[7] Deenen, J. and Quesne, C. (1985) Boson Representations of the Real Symplectic Group and Their Applications to the Nuclear Collective Model. Journal of Mathematical Physics, 26, 2705-2716.

[8] Roy, A.K. (2016) Critical Parameters and Spherical Confinement of H Atom in Screened Coulomb Potential. International Journal of Quantum Chemistry, 116, 953-960. https://doi.org/10.1002/qua.25108

[9] Ita, B.I. and Ekuri, P. (2010) Bound State Solutions of Schrodinger Equation for a More General Exponential Screened Coulomb Potential via Nikiforov-Uvarov Method. Ecleticalquimica, 35, 103-107. https://doi.org/10.1590/S0100-46702010000300009

[10] Yukawa, H. (1935) On the Interaction of Elementary Particles. Proceedings of the Physico-Mathematical Society, 17, 48-57.

[11] Gerry, C.C. and James, B.T. (1986) A Large-N Phase Integral Approximation for Coulomb-Type System using SO $(2,1)$ Coherent States. Journal of Physics A, 19, 3797-3808. https://doi.org/10.1088/0305-4470/19/18/025

[12] Hamzavi, M., Ikhdair, S.M. and Sulaimani, M. (2012) A Semirelativistic Treatment of Spinless Particles Subject to the Yukawa Potential with Arbitrary Angular Momenta. International Journal of Modern Physics E, 21, 1-13. https://doi.org/10.1142/S0218301312500164

[13] Dutt, R., Chowdhury, R. and Varshni, Y.P. (1985) An Improved Calculation for Screened Coulomb Potential in Rayleigh-Schrodinger Perturbation Theory. Journal of Physics A: Mathematical and General, 18, 1379-1388.

[14] Hamzavi, M., Movahedi, M., Thylwe, K.E. and Rajabi, A.A. (2012) Approximate Analytic Solutions of the Yukawa Potential with Arbitrary Angular Momenta. Chemical Physics Letters, 29, 1-4. 
[15] Gerry, C.C. and James, B.T. (1986) Generalized Scaling Variational Method and Energy Eigenvalues for the Yukawa Potential. Physical Review A, 32, 709-712. https://doi.org/10.1103/PhysRevA.32.709

[16] Soylu, A., Bayrak, O. and Boztosun, I. (2008) Exact Solutions of the Klein-Gordon Equation with Equal Scalar and Vector Rosen-Morse Type Potential. Chinese Physics Letters, 25, 2754-2757. https://doi.org/10.1088/0256-307X/25/8/006

[17] Pakdel, F., Rajabi, A.A. and Hamzavi, M. (2014) Scattering and Bound State Solutions of the Yukawa Potential within the Dirac Equation. Advances in High Energy Physics, 2014, Article ID: 867483.

[18] Roy, A.K. (2013) Studies on Some Exponential-Screened Coulomb Potential. International Journal of Quantum Chemistry, 113, 1503-1510. https://doi.org/10.1002/qua.24351

[19] Onate, C.A. and Ojonubah, J.O. (2016) Eigensolutions of the Schrödinger Equation with a Class of Yukawa Potential via Supersymmetric Approach. Journal of Theoretical and Applied Physics, 10, 21-26. https://doi.org/10.1007/s40094-015-0196-2

[20] Ita, B.I., Louis, H., Magu, T.O. and Nzeata-Ibe, N.A. (2017) Bound State Solutions of the Schrödinger Equation with Manning-Rosen plus a Class of Yukawa Potential using Pekeris-Like Approximation of the Coulomb Term and Parametric Nikiforov-Uvarov Method. World Scientific News, 70, 267-385.

[21] Cooper, F., Khare, A. and Sukhatme, U. (1995) Supersymmetry and Quantum Mechanics. Physics Reports, 251, 267-385.

https://doi.org/10.1016/0370-1573(94)00080-M

[22] Chen, G. (2004) Shape Invariance and the Supersymmetric WKB Approximation for the Generalized Hulthen Potential. Physica Scripta, 69, 257-259. https://doi.org/10.1238/Physica.Regular.069a00257

[23] Nikiforov, A.F. and Uvarov, V.B. (1988) Special Mathematical Functions of Mathematical Physics. https://doi.org/10.1007/978-1-4757-1595-8

[24] Louis, H., Ita, B.I., Nelson, N.A., Joseph, I., Amos, P.I. and Magu, T.O. (2017) Wkb Solutions for Quantum Mechanical Gravitational Potential plus Harmonic Oscillator Potential. International Research Journal of Pure and Applied Physics, 5, 27-32.

[25] Hitler, L., Ita, B.I., Isa, P.A., Nelson, N.-I., Joseph, I., Ivan, O. and Magu, T.O. (2017) Wkb Solutions for Inversely Quadratic Yukawa plus Inversely Quadratic Hellmann Potential. World Journal of Applied Physics, 2, 109-112.

[26] Hitler, L., Ita, B.I., Isa, P.A., Joseph, I., Nelson, N.-I., Magu, T.O. and Ivan, O. (2017) Analytic Spin and Pseudospin Solutions to the Dirac Equation for the Manning-Rosen plus Hellmann Potential and Yukawa-Like Tensor Interaction. World Journal of Applied Physics, 2, 101-108.

[27] Ita, B.I., Ikeuba, A.I., Louis, H. and Tchoua, P. (2015) Solutions of the Schrodinger Equation with Inversely Quadratic Yukawa Plus Attractive Radial Potential Using Nikiforov-Uvarov Method. Journal of Theoretical Physics and Cryptography, 10.

[28] Ita, B.I., Louis, H., Amos, P.I., Joseph, I., Nzeata-Ibe, N.A., Magu, T.O. and Disho, H. (2017) L-State Analytical Solution of the Klein-Gordon Equation with Position Dependent Mass using Modified Deng-Fan plus Exponential Molecular Potentials via Nikiforov-Uvarov Method. World Scientific News, 89, 64-70.

[29] Ita, B.I., Louis, H., Amos, P.I., Magu, T.O. and Nzeata-Ibe, N.A. (2017) Bound State Solutions of the Klein-Gordon Equation with Manning-Rosen plus Yukawa Potential using Pekeris-Like Approximation of the Coulomb Term and Parametric Niki-forov-Uvarov. Physical Science International Journal, 15, 1-6. 
[30] Nelson, N.-I., Ita, B.I., Joseph, I., Isa, P.A., Magu, T.O. and Hitler, L. (2017) Analytic Spin and Pseudospin Solutions to the Dirac Equation for the Quadratic Exponential-Type Potential plus Eckart Potential and Yukawa-Like Tensor Interaction. World Journal of Applied Physics, 2, 77-84.

[31] Ikhdair, S.M. (2011) Bound State of the Klein-Gordon for Exponential-Type Potential in D-Dimensions. Journal of Quantum Information Science 1, 73-86.

[32] Ikot, A.N., Obon, H.P., Abbey, T.M. and Olisa, J.D. (2015) Approximate Analytical Solutions of Klein-Gordon Equation with an Exponential-Type Potential. Sae Mulli: New Physics, 65, 825-836.

[33] Hassanbadi, H., Rahimov, H. and Zarrinkamar, S. (2011) Approximate Solutions of Klein-Gordon Equation with Kratzer Potential. Advances in High Energy Physics, 2011, Article ID: 458087.

[34] Ikot, A.N., Udoimuk, A.B. and Akpabio, I.E. (2011) Bound State Solutions of Klein-Gordon Equation with Type-l-equal Scalar and Vector Poschl-Teller Potential for Arbitrary 1-State. American Journal of Scientific and Industrial Research, 2, 179-183.

[35] Lu, J. (2005) Analytic Quantum Mechanics of Diatomic Molecules with Empirical Potentials. Physics Scripta, 72, 349-352. https://doi.org/10.1238/Physica.Regular.072a00349

[36] Greene, R.L. and Aldrich, C. (1976) Variational Wave Functions for a Screened Coulomb Potential. Physical Review A, 14, 2363-2366. https://doi.org/10.1103/PhysRevA.14.2363

[37] Jia, C.S., Chen, T. and Cui, L.G. (2009) Approximate Analytical Solutions of the Dirac Equation with the Generalized Pöschl-Teller Potential Including the Pseudo-Centrifugal Term. Physics Letters A, 373, 1621-1626.

[38] Hill, E.H. and Am, J. (1954) The Theory of Vector Spherical Harmonics. American Journal of Physics, 22, 211.

[39] Karplus, M. and Porter, R.N. (2007) Atoms and Molecules: An Introduction for Students of Physical Chemistry. Benjamin-Cummings Pub. Co., MenloPark. 